\title{
Extensive deployment of the stented elephant trunk is associated with an increased risk of spinal cord injury
}

Jorge Flores, MD, PhD, Takashi Kunihara, MD, PhD, Norihiko Shiiya, MD, PhD, Kimihiro Yoshimoto, MD, PhD, Kenji Matsuzaki, MD, and Keishu Yasuda, MD, PhD

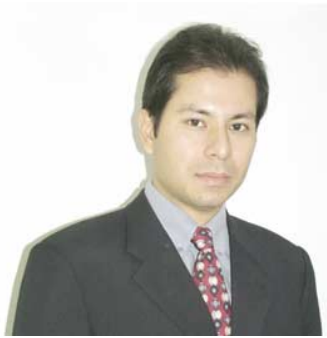

Dr Flores

See related editorial on page 261.
From the Department of Cardiovascular Surgery, Hokkaido University Graduate School of Medicine, Hokkaido, Japan.

Received for publication July 7, 2005; revisions received Sept 2, 2005; accepted for publication Sept 15, 2005.

Address for reprints: Takashi Kunihara, MD, $\mathrm{PhD}$, Department of Cardiovascular Surgery, Hokkaido University Graduate School of Medicine, N14W5, Kita-Ku, Sapporo, Hokkaido, Japan 060-8648 (E-mail: kunihara@ med.hokudai.ac.jp).

J Thorac Cardiovasc Surg 2006;131:336-42

$0022-5223 / \$ 32.00$

Copyright $\odot 2006$ by The American Association for Thoracic Surgery

doi:10.1016/j.jtcvs.2005.09.050
Objective: Thoracic aortic aneurysm repair with the stented elephant trunk technique seems to be associated with an increased risk of spinal cord injury. We investigated whether severe atherosclerosis of the distal landing zone or extensive deployment of the stented elephant trunk is associated with increased risk of spinal cord injury.

Methods: Twenty-five patients underwent thoracic aortic aneurysm repair with the stented elephant trunk technique. The study population included 19 men and had a mean age of $73 \pm 7$ years. All patients underwent a median sternotomy with cardiopulmonary bypass and selective cerebral perfusion. The elephant trunk was fixed with a Z-stent distal to the aneurysm during hypothermic circulatory arrest. Thirteen patients underwent concomitant total aortic arch replacement.

Results: Six (24\%) patients had spinal cord injury. The presence of severe atherosclerosis at the distal landing zone demonstrated a tendency to increase the incidence of spinal cord injury (36\% vs $9 \%, P=.1218$ ). More distal deployment of the stented elephant trunk was significantly associated with increased risk of spinal cord injury (T8.0 \pm 0.6 vs T6.5 $\pm 1.1, P=.0043)$. Univariate logistic regression analysis identified a history of abdominal aortic aneurysm repair $(P=.0296)$ and the vertebral level of the distal landing zone $(P=.0249)$ as significant independent risk factors for spinal cord injury, and only the latter was significant in multivariate analysis $(P=.0396)$. The combination of a distal landing zone of $\mathrm{T} 7$ or greater and a history of abdominal aortic aneurysm repair was the strongest predictor for spinal cord injury $(71 \%$ vs $6 \%, P=.0047)$.

Conclusions: Spinal cord injury after stented elephant trunk deployment might be related to occlusion of the excessive intercostal arteries or thromboembolism. Patients with a history of abdominal aortic aneurysm repair who require extensive deployment of the stented elephant trunk seem to be at a higher risk for spinal cord injury.

$\mathrm{T}$ horacic aortic aneurysm repair through a median sternotomy with deployment of a stent graft distal to the aneurysmal sac in a fashion similar to the elephant trunk technique ${ }^{1}$ has been described as a good option of treatment, especially in patients with high-risk conditions. ${ }^{2-8}$ This type of operation, usually termed repair by the stented (or "frozen") elephant trunk (SET) technique, ${ }^{9-11}$ can simplify aortic reconstruction and reduce operative time, bleeding, and respiratory morbidity by avoiding a left thoracotomy. In spite of these advantages, spinal cord injury (SCI) has emerged as an unacceptable cause of morbidity after this type of operation. The incidence of SCI after SET repair seems considerably higher than one might expect, fluctuating from $4.5 \%$ to $12.5 \%$ in published literature covering more than 10 cases. ${ }^{7,8,10,11}$ However, the underlying mechanisms of this unpredictable complication are still not understood. 

Abbreviations and Acronyms
AAA $=$ abdominal aortic aneurysm
COPD $=$ chronic obstructive pulmonary disease
$\mathrm{CT}=$ computed tomography
SCI = spinal cord injury
SET $=$ stented elephant trunk

Svensson and colleagues ${ }^{1}$ recommended that the elephant trunk graft should be shorter than 10 to $15 \mathrm{~cm}$ from their experiences with 3 patients with SCI after deployment of a long elephant trunk. Chavan and coworkers ${ }^{11}$ also recommended that the distal landing zone of the stent graft should be proximal to the 10th thoracic vertebral level for possible reduction in the incidence of SCI. Therefore we can speculate that the excessive occlusion of the intercostal arteries that augment the spinal cord blood flow might be responsible for SCI. Alternatively, thromboembolism to the intercostal arteries that might be provoked by insertion and deployment of SET might be involved in the mechanism of SCI. Recent advances in radiologic methods of aortic evaluation highlighted the relation between the presence of atheromatous plaques in the aortic wall and the probability of postoperative neurologic injury. Tenenbaum and associates, ${ }^{12}$ who evaluated the aortic arch in addition to the thoracic descending aorta, have reported that a calcium deposit or clearly visualized area of hypoattenuation at least $4 \mathrm{~mm}$ thick adjacent to the aortic wall might become a source of iatrogenic thromboembolism and stroke. For the spinal cord, however, neither clinical nor experimental confirmation of this hypothesis in SET repair has been reported to date.

In this work we present our experience in the management of 25 patients with thoracic aortic aneurysm who were treated by using the SET technique and assess whether postoperative SCI can be predicted by the presence of severe atherosclerosis or by the level of stent graft deployment.

\section{Methods}

\section{Patients' Characteristics}

From January 1996 through December 2004, 25 patients with thoracic aortic aneurysms underwent replacement of the diseased segment by means of SET repair. Inclusion criteria for this type of operation were related to elderly patients with multiple concomitant diseases, the presence of severe chronic obstructive pulmonary disease (COPD), the presence of a mega-aorta that must be repaired in one stage, the presence of severe atheromatous disease around the distal aneurysmal neck, and previous surgical intervention on the thoracic aorta. Patients with the same diagnosis but in relatively good clinical condition and without previous aortic surgery were selected to undergo conventional graft replacement of the diseased segment.

There were $19(76 \%)$ male and $6(24 \%)$ female patients. The mean age was $73 \pm 7$ years (range, $54-83$ years). Preoperative risk factors included previous cerebrovascular accident in $11(44 \%)$ patients, hypertension in $21(84 \%)$ patients, ischemic heart disease in $6(24 \%)$ patients, diabetes mellitus in $2(8 \%)$ patients, COPD in $11(44 \%)$ patients, and a history of smoking in $20(80 \%)$ patients. Renal dysfunction, defined as a serum creatinine level of $2 \mathrm{mg} / \mathrm{dL}$ or greater, was present in $3(12 \%)$ patients.

Eight $(32 \%)$ patients had a history of thoracic aortic repair. Only one of them was operated on through a median sternotomy for total arch replacement. Five of the patients underwent descending thoracic artery aneurysm repair, and the other 2 patients underwent thoracoabdominal aortic aneurysm repair. Ten (40\%) patients had a history of infrarenal abdominal aortic aneurysm (AAA) repair, and one of them had a history of postoperative transient right lower leg monoparesis (this patient also had left lower leg monoparesis after SET repair).

Twenty-three (92\%) patients underwent elective surgical repair of the proximal descending aorta by using the SET technique, and $2(8 \%)$ patients were operated on in an emergency setting. There were $13(52 \%)$ patients with concomitant aortic arch aneurysms, including one case of chronic DeBakey type I aortic dissection that became aneurysmal. In the other $12(48 \%)$ patients, the aneurysm was located exclusively distal to the left subclavian artery. In 3 of these patients, anastomotic pseudoaneurysms developed after previous thoracic aortic repair. Two cases of proximal descending aortic aneurysm evolved to rupture, including 1 patient with acute DeBakey type IIIa aortic dissection. Associated operations included coronary artery bypass grafting in $3(12 \%)$ patients, mitral valvuloplasty in 1 (4\%) patient, and bypass grafting to the left subclavian artery in $1(4 \%)$ patient.

The cause was atherosclerosis in $18(72 \%)$ patients, chronic dissection in 5 (20\%) patients, medial cystic necrosis in $1(4 \%)$ patient, and Takayasu's disease in $1(4 \%)$ patient.

\section{Surgical Technique}

The stent graft for thoracic aortic aneurysm repair by means of the SET technique is assembled from a 5-cm-long Gianturco stent (Cook, Bloomington, Ind) inserted into the distal segment of a Hemashield Gold graft (Boston Scientific, Natick, Mass) and fixed to it by interrupted stitches of polyester thread. The size of the graft depends on the length of the aneurysmal sac and the diameter of the landing zone at the aorta distal to the aneurysmal sac. This is determined by means of preoperative 3-dimensional computed tomography (CT). The diameter of the graft should be $110 \%$ of the diameter of the aortic segment chosen as the landing zone. The diameter of the stent depends on the graft diameter. A stent $40 \mathrm{~mm}$ in diameter is used for a 30-mm or larger graft, whereas a $30-\mathrm{mm}$ stent is used for a 28 -mm or smaller graft. The stent graft is then bound to a curved tube by a chain stitch. ${ }^{13}$

Our surgical protocol for the SET technique is the same protocol that we routinely use in cases of conventional aortic arch repair and has been previously described. ${ }^{14,15}$ An arterial cannula is placed in the proximal ascending aorta or the axillary artery (left, 5; right, 1; bilateral, 3) in case the ascending aorta is not suitable for cannulation. All 3 arch vessels are routinely perfused antegradely. After induction of circulatory arrest of the lower torso when the bladder temperature reaches $22^{\circ} \mathrm{C}$, the aortic arch is transected. The stent graft is inserted into the descending thoracic aorta distal to the aneurysmal sac (Figure 1,A). The chain stitch is 

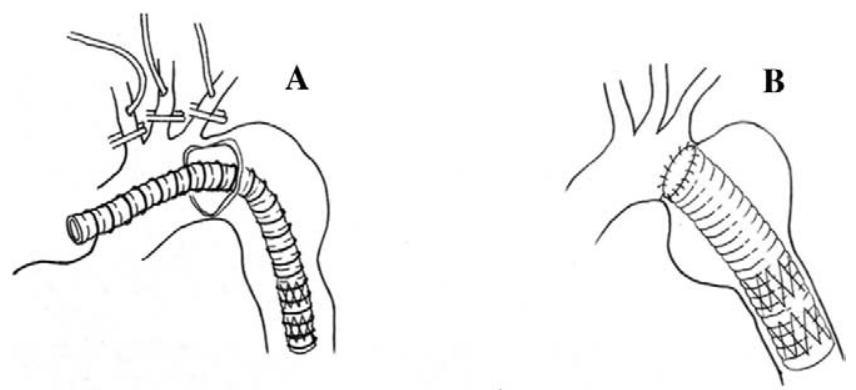

Figure 1. Schema of thoracic aortic aneurysm repair by means of the stented elephant trunk (SET) technique: A, SET deployment after opening of the aortic aneurysmal sac; B, repair of a descending thoracic aortic aneurysm by means of the SET technique; C, concomitant reconstruction of an aortic arch and descending thoracic aortic aneurysm by means of the SET technique.

released, and then the stent graft is expanded and fixed to the aortic wall by a balloon catheter advanced and inflated at the distal attachment site. No fluoroscopic monitoring is used during the deployment. The proximal end of this trunk is anastomosed to the proximal neck of the aneurysmal sac (Figure 1, B). When total arch replacement is necessary, a commercially available 4-branched Gelseal (Sulzer Vascutek, Renfrewshire, Scotland) prosthesis is attached to the proximal end of the SET by continuous suturing with 4-0 polypropylene sutures, which includes the aneurysmal wall. After distal anastomosis, a clamp is placed on the branched aortic prosthesis, systemic blood perfusion is resumed through the fourth side branch, and the patient is gradually rewarmed. Proximal aortic anastomosis is then carried out, and coronary blood flow is restored. The remaining 3 branch grafts are anastomosed to the 3 arch vessels in an end-to-end fashion, and selective cerebral perfusion is discontinued (Figure 1,C). Concomitant operations, such as coronary artery bypass grafting, can be performed during either the cooling or rewarming period.

\section{Postoperative Examination}

The level of deployment of the distal end of the SET was checked by means of chest radiography. All patients were subjected to postoperative neurologic examination, and brain CT was indicated if a neurologic deficit was detected.

All patients underwent 3-dimensional CT to evaluate the proximal anastomosis and distal attachment site of the stent graft. Information regarding long-term follow-up was obtained during examination of patients at the outpatient clinic or by telephone interview with the patients or their relatives.
The aortic wall quality and the level at which the SET was landed were assessed by means of preoperative CT according to the criteria of Tenenbaum and associates ${ }^{12}$ and postoperative chest radiography, respectively, to predict the possibility of postoperative SCI.

\section{Statistical Analysis}

All values are expressed as means \pm standard deviation. Statistical analysis was performed with the Statview 5.0 program (SAS Institute Inc, Cary, NC). The Student $t$ test was used for comparison of the continuous variables, and a $\chi^{2}$ test was used for comparison of frequencies between the groups. Survival was estimated by using the Kaplan-Meier method. By using logistic regression, preoperative, intraoperative, and postoperative variables were analyzed to identify risk factors for SCI (see Appendix 1 for variables evaluated). Then a $P$ value of less than .10 was defined for selecting variables for entry into the multivariate model.

\section{Results}

\section{Spinal Cord Injury}

Ischemic SCI was present in $6(24 \%)$ patients who experienced lower limb paraparesis or monoparesis, 3 patients each, including 1 patient with left lower limb monoparesis who had a concomitant stroke. This patient had multiple small cerebral infarctions at the bilateral cerebellum, the bilateral occipital lobe, and the left temporal lobe that did not seem to be responsible for his left lower limb monoparesis. The postoperative CT scan did not provide evidence of fresh intracranial brain injury in the remaining patients with monoparesis. Two patients with monoparesis experienced total recovery.

The distal end of the stent graft was deployed at the T5 level in 3 patients, at the T6 level in 7 patients, at the T7 level in 7 patients, at the T8 level in 6 patients, and at the T9 level in 2 patients. In the group of 6 patients who presented with postoperative SCI, the SET was deployed at the T7 level in 1 patient, at the T8 level in 4 patients, and at the T9 level in 1 patient (Figure 2$)$. There was a significant difference $(P=$ .0043) regarding the mean value of the thoracic vertebral level, where the distal end of the SET was deployed between those patients with $($ T8.0 \pm 0.6$)$ and without (T6.5 \pm 1.1) SCI.

According to the criteria of Tenenbaum and coworkers, ${ }^{12}$ severe atherosclerosis was found in the SET landing zone in 14 patients in our series; among them, $5(36 \%)$ patients had postoperative SCI. In the remaining 11 patients, only $1(9 \%)$ had postoperative SCI. This patient underwent an emergency operation for rupture. The difference between the incidence of SCI among patients with or without severe atherosclerosis was not significant ( $P=.1218$, Figure 3 ).

Univariate logistic regression analysis identified vertebral level of distal landing zone $(P=.0294)$ and history of AAA repair $(50.0 \%$ vs $6.7 \%, P=.0296$, Figure 4$)$ as the 


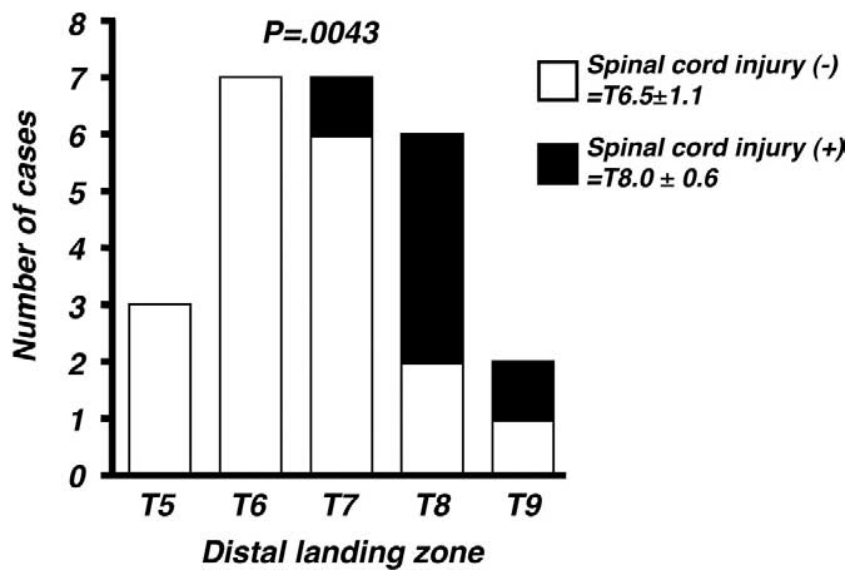

Figure 2. Relationship between postoperative spinal cord injury and level of stented elephant trunk deployment at the descending aorta. T, Thoracic vertebral level. Open bar graphs, Patients without spinal cord injury; filled bar graphs, patients with postoperative spinal cord injury.

significant independent risk factors for SCI. Multivariate logistic regression analysis identified only the former as a significant independent risk factor for SCI $(P=.0396$; odds ratio, 5.462; 95\% confidential interval, 1.084-27.514). When distal landing zone at $\mathrm{T} 7$ or a more distal point and history of AAA repair were combined, this was the strongest significant independent predictor for SCI $(71.4 \%$ vs $5.6 \% ; P=.0047$; odds ratio, $5.462 ; 95 \%$ confidence interval, 1.084-571.963; Table 1 and Figure 5; see Appendix 1 for variables evaluated).

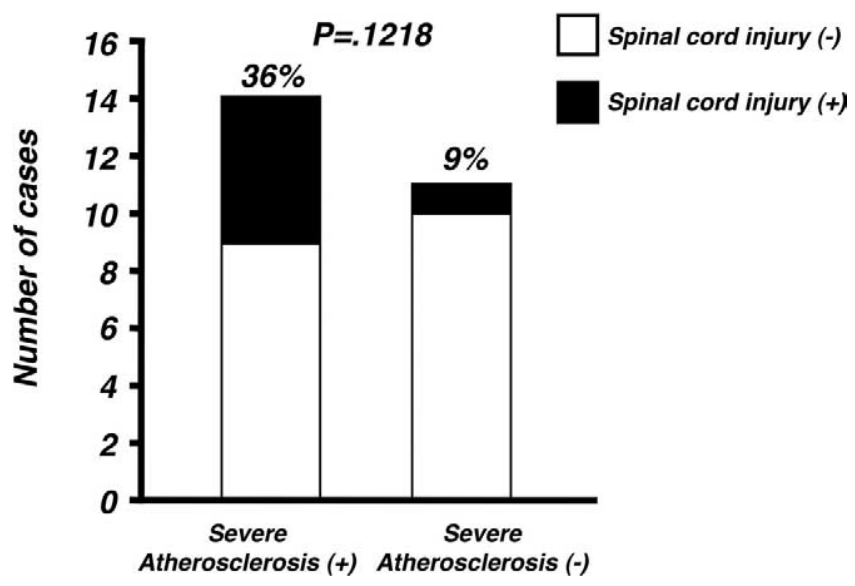

Figure 3. Relationship between postoperative spinal cord injury and atherosclerosis of the descending thoracic aorta for distal landing zone. Open bar graphs, Patients without spinal cord injury; filled bar graphs, patients with postoperative spinal cord injury.
Other Postoperative Complications

There were 3 (12\%) hospital deaths. One patient died on the 29th postoperative day after an anaphylactic shock against contrast media during a CT scan. One octogenarian patient who had severe COPD died on the 24th postoperative day because of respiratory failure. The other patient died from a hemorrhagic shock after rupture of his descending aortic aneurysm while waiting for a second operation because the SET was deployed shorter than planned. This was the only death directly related to SET repair. There were $4(16 \%)$ cases of stroke and $2(8 \%)$ cases of left recurrent nerve injury. Prolonged ventilatory support ( $>72$ hours) was required in 3 patients.

\section{Long-Term Follow-up}

The mean follow-up was $35 \pm 13$ months (range, 2-48 months). During the follow-up, 4 patients died. One patient died from unknown cause 20 months after the operation. Another patient died after AAA repair in another institute 14 months after our operation. The other 2 patients died from pneumonia in postoperative months 17 and 44, respectively. Actuarial survival was $70 \%$ at 2 years and $60 \%$ at 4 years (Figure 6).

\section{Discussion}

Although recent advances in the surgical treatment of aortic arch disease have achieved acceptable outcomes, there remains a higher rate of mortality and morbidity in patients with extensive disease and high-risk conditions in relation to comorbidities. ${ }^{16,17}$ Since the report of the pioneer experiences in the management of these high-risk subgroups of patients with SET repair in 1996, ${ }^{2,3}$ several works that mention the advantages of this technique in comparison with conventional graft replacement or endovascular surgery have been published. ${ }^{4-8,9-11}$ In the case of aneurysm or

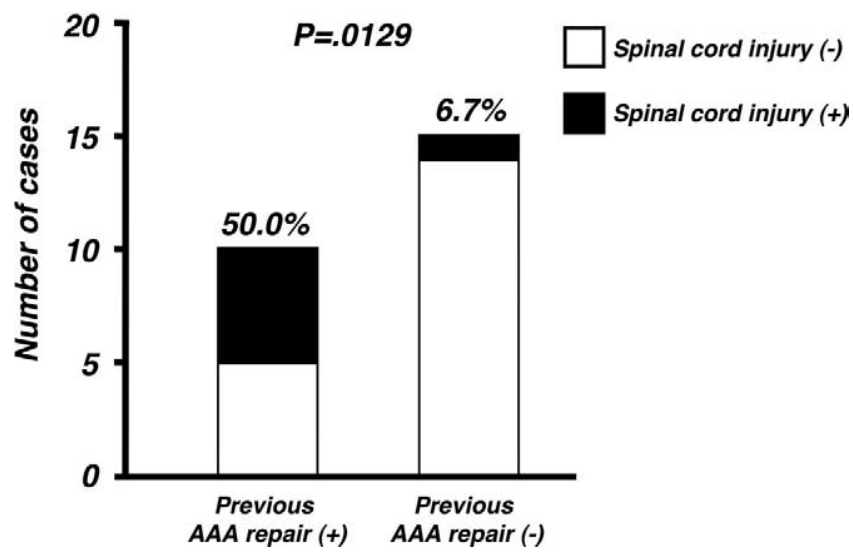

Figure 4. Relationship between postoperative spinal cord injury and history of previous abdominal aortic aneurysm repair. Open bar graphs, Patients without spinal cord injury; filled bar graphs, patients with postoperative spinal cord injury. 
TABLE 1. Univariate and multivariate logistic regression analysis to identify risk factors for spinal cord injury

\begin{tabular}{|c|c|c|c|c|c|c|c|}
\hline \multirow[b]{2}{*}{ Variable } & \multirow[b]{2}{*}{ SCI } & \multicolumn{3}{|c|}{ Univariate } & \multicolumn{3}{|c|}{ Multivariate } \\
\hline & & $P$ value & $\mathbf{O R}$ & $95 \%$ CI & $P$ value & $\mathbf{O R}$ & $95 \%$ Cl \\
\hline Distal landing zone level & $\begin{array}{l}\mathrm{SCl}(+): \mathrm{T} 8.0 \pm 0.6 \\
\mathrm{SCl}(-): \mathrm{T} 6.5 \pm 1.1\end{array}$ & .0249 & 5.153 & $1.23-21.591$ & .0396 & 5.462 & $1.084-27.514$ \\
\hline History of AAA repair & $\begin{array}{l}\text { Yes: SCI }=5 / 10(50.0 \%) \\
\text { No: } \mathrm{SCI}=1 / 15(6.7 \%)\end{array}$ & .0296 & 14 & $1.299-150.924$ & .0606 & 18.272 & $0.879-379.876$ \\
\hline $\begin{array}{c}\text { Distal landing zone level } \geq T 7 \\
\text { and history of AAA repair }\end{array}$ & $\begin{array}{l}\text { Yes: SCI = 5/7 (71.4\%) } \\
\text { No: } \mathrm{SCI}=1 / 18(5.6 \%)\end{array}$ & .0047 & 5.462 & $1.084-571.963$ & & & \\
\hline
\end{tabular}

$S C l$, Spinal cord injury; $O R$, odds ratio; $C l$, confidence interval; $A A A$, abdominal aortic aneurysm.

dissection extending from the distal arch into the distal descending aorta, endovascular deployment of a stent graft is usually a difficult maneuver. Conventional prosthesis replacement can be performed through a sternotomy, thoracotomy, or combination of both. However, distal exposure is not optimal through a sternotomy alone, and the risk of stroke is high through a thoracotomy alone in the presence of severe atheromatous disease. One of the benefits of SET repair, particularly in the case of a previous left thoracotomy, is the avoidance of rethoracotomy, which might present the potential risks of excessive bleeding, prolonged surgical time, and respiratory failure. Furthermore, severe adhesion might prevent adequate mobilization of the distal anastomosis site from a midline sternotomy approach. In all of our patients, left thoracotomy and lung compression, which are hazardous to patients with COPD, were avoided. Stent graft fixation to the descending aortic wall can avoid creation of a distal hand-sewn anastomosis, which can be a difficult maneuver through a sternotomy. When severe atheromatous disease exists around the distal aneurysmal neck, a conventional hand-sewn anastomosis might entail the

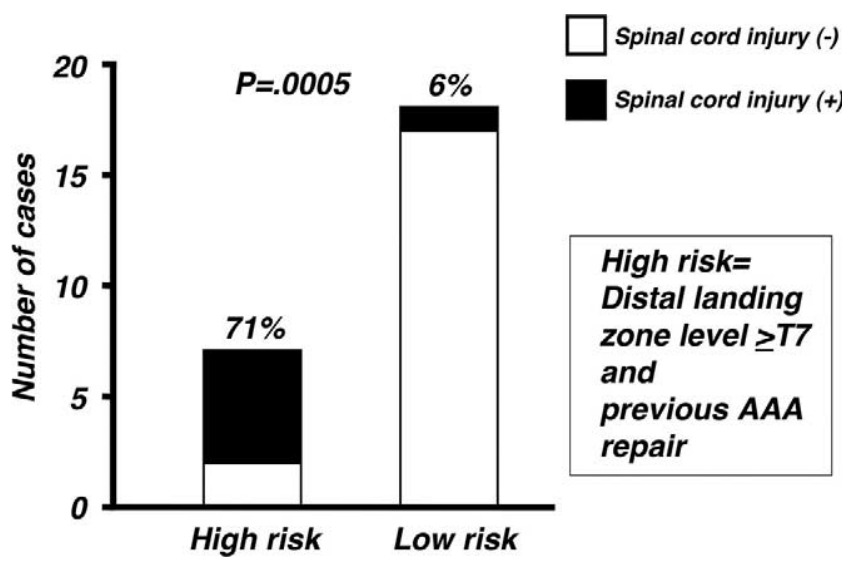

Figure 5. Relationship between postoperative spinal cord injury and combination of distal landing zone level at T7 or greater and history of previous abdominal aortic aneurysm repair. Open bar graphs, Patients without spinal cord injury; filled bar graphs, patients with postoperative spinal cord injury. great risks of not only massive distal embolization but also excessive bleeding. Furthermore, the proximal anastomosis is performed by means of conventional suture at a level at which endovascular stent graft attachment would be hazardous.

In spite of the benefits mentioned above, this procedure was associated with a high incidence of SCI. From our analysis, the underlying mechanism might be excessive sacrifice of intercostal arteries or thromboembolism. Some cases of postoperative SCI might be related to occlusion of the critical intercostal arteries that perfused the spinal cord by the distal end of the stent grafts. However, it is known that the Adamkiewicz artery enters the vertebral canal between $\mathrm{T} 9$ and $\mathrm{T} 12$ in $76 \%$ of cases and between $\mathrm{T} 7$ and $\mathrm{T} 8$ in $12 \%$ of cases. ${ }^{18,19}$ In our group only 2 patients underwent SET repair extended distally to the T9 level. Therefore it seems reasonable to speculate that extensive sacrifice of intercostal arteries that do not directly supply spinal cord blood flow but augment collateral blood flow might be an underlying mechanism predisposing to SCI. Svensson and colleagues ${ }^{1}$ or Chavan and coworkers ${ }^{11}$ also recommended that extensive deployment of the elephant trunk or SET should be avoided to reduce potential risk of postoperative

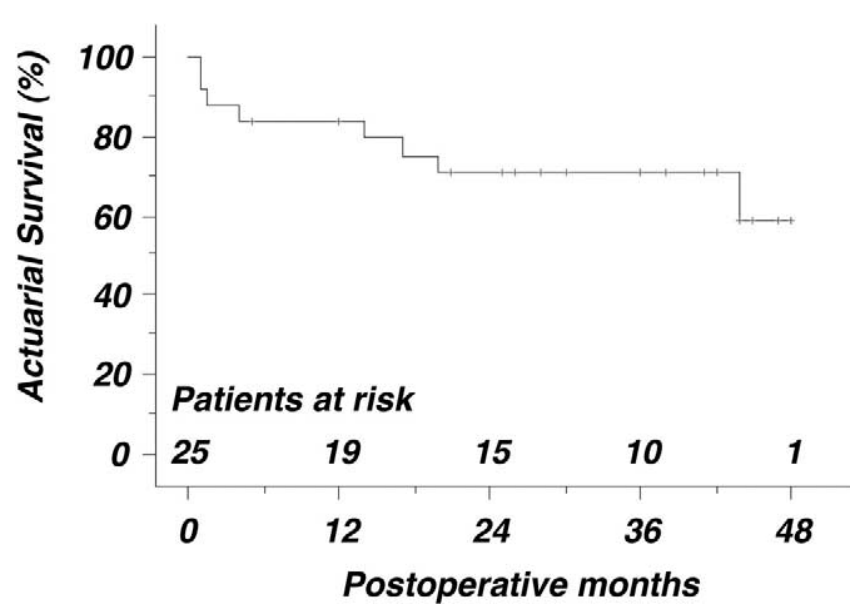

Figure 6. Kaplan-Meier survival curve after thoracic aortic aneurysm repair by means of the stented elephant trunk technique. 
SCI. This speculation was supported by the fact that the risk of SCI was even higher when the SET was deployed extensively in patients with a history of previous AAA repair. Even in the endovascular approach alone, it has been reported that preventive cerebrospinal fluid drainage has been used to reduce the risk of SCI for patients with a history of previous AAA repair. ${ }^{20}$

Another mechanism might include atheroembolism to the spinal cord. ${ }^{12,21,22}$ Although the difference between the incidence of SCI among patients with or without severe atherosclerosis was not significant, there was a tendency for development of SCI in the former group (36\% vs 9\%, Figure 3). Except for the case of rupture, the remaining 5 patients with postoperative SCI reported in this series presented with protruding atherosclerotic lesions located at the descending aorta distal to the aneurysmal sac that might be dislodged during stent graft deployment or subsequent to the re-establishment of the aortic blood flow. This speculation is strongly supported by a report from Usui and coworkers. ${ }^{10}$ In their 3 patients with postoperative SCI after SET repair, the spinal cord was injured at a level remote from the SET landing zone (lower thoracic, fourth-fifth lumbar, and sacral level), which suggested that an embolic event was responsible for their SCI.

Compared with other alternatives of treatment of thoracic aortic aneurysm, such as endovascular repair, in which the blood flow is not interrupted and the systolic pressure is maintained around its normal value throughout the procedure, ${ }^{23}$ the SET technique involves cardiac arrest during deep hypothermia and temporary interruption of systemic blood perfusion during the distal deployment. The inflammatory response to cardiopulmonary bypass and the free radicals originating in the ischemia-reperfusion injury lead to increased capillary pressure and microvascular permeability, producing fluid extravasation in several organs, including the spinal cord. ${ }^{24,25}$ Because the spinal cord sheath, the dura, and the surrounding bony vertebral channel are nondistensible, the spinal cord is subjected to a compartment syndrome,${ }^{26}$ which could increase the possibility of SCI. Cerebrospinal fluid drainage might attenuate this negative effect of cardiopulmonary bypass, but at our department, we do not use this method in cases of deep hypothermic circulatory arrest because of the potential risk of intracranial hemorrhage. ${ }^{27}$ In addition, we used no fluoroscopic monitoring during SET deployment. Therefore the SET was apt to be deployed more distally than when done precisely through the endovascular approach. In another alternative, such as delayed (staged) open surgical repair with a conventional elephant trunk technique, ${ }^{1}$ one can reattach the intercostal arteries if necessary. The conventional elephant trunk technique does not manipulate the distal aneurysmal neck, which is a potential source of distal embolization when severe atheromatous disease exists. This might be the reason that the SET technique has a greater risk of SCI than delayed (staged) open surgical or endovascular repair.

\section{Conclusions}

In our relatively elderly patients with several comorbidities, the incidence of SCI after SET repair seemed considerably higher than one might expect. The underlying mechanism might be excessive sacrifice of intercostal arteries that jeopardizes collateral blood flow to the spinal cord or thromboembolic event. A history of previous AAA repair was also an increased risk for SCI. Therefore patients with a history of AAA repair who might require extensive deployment of an SET should be managed by other alternatives to avoid postoperative SCI.

We thank Yasuaki Saijo, MD, PhD, Department of Public Health, Hokkaido University Graduate School of Medicine, for his advice for statistical analysis.

\section{References}

1. Svensson LG, Kim KH, Blackstone EH, Alster JM, McCarthy PM, Greenberg RK, et al. Elephant trunk procedure: newer indications and uses. Ann Thorac Surg. 2004;78:109-16.

2. Kato M, Ohnishi K, Kaneko M, Ueda T, Kishi D, Mizushima T, et al. New graft-implanting method for thoracic aortic aneurysm or dissection with a stented graft. Circulation. 1996;94(suppl II):II188-93.

3. Suto Y, Yasuda K, Shiiya N, Murashita T, Kawasaki M, Imamura M, et al. Stented elephant trunk procedure for an extensive aneurysm involving distal aortic arch and descending aorta. J Thorac Cardiovasc Surg. 1996;112:1389-90.

4. Okada K, Sueda T, Kazumasa O, Watari M, Ishii O. An alternative procedure of endovascular stent-graft repair for distal arch aortic aneurysm involving arch vessels. J Thorac Cardiovasc Surg. 2001; 121:182-4.

5. Sueda T, Watari M, Okada K, Orihashi K, Matsuura Y. Endovascular stent-grafting through the aortic arch: an alternative approach for distal arch aortic aneurysm. Ann Thorac Surg. 2000;70:1251-4.

6. Mizuno T, Toyama M, Tabuchi N, Wu H, Sunamori M. Stented elephant trunk procedure combined with ascending aorta and arch replacement for acute type A aortic dissection. Eur J Cardiothorac Surg. 2002;22:504-9.

7. Kato M, Kuratami T, Kaneko M, Kyo S, Ohnishi K. The results of total arch graft implantation with open stent-graft placement for type A aortic dissection. J Thorac Cardiovasc Surg. 2002;124:531-40.

8. Uchida N, Ishihara H, Sakashita M, Kanou M, Sumiyoshi T. Repair of the thoracic aorta by transaortic stent grafting (open stenting). Ann Thorac Surg. 2002;73:444-9.

9. Karck M, Chavan A, Khaladj N, Friedrich H, Hagl C, Haverich A. The frozen elephant trunk technique for the treatment of extensive thoracic aortic aneurysms: operative results and follow-up. Eur J Cardiothorac Surg. 2005;28:286-90.

10. Usui A, Fujimoto K, Ishiguchi T, Yoshikawa M, Akita T, Ueda Y. Cerebrospinal dysfunction after endovascular stent-grafting via a median sternotomy: the frozen elephant trunk procedure. Ann Thorac Surg. 2002;74(suppl):S1821-4.

11. Chavan A, Karck M, Hagl C, Winterhalter M, Baus S, Galanski M, et al. Hybrid endograft for one-step treatment of multisegment disease of the thoracic aorta. $J$ Vasc Interv Radiol. 2005;16:823-9.

12. Tenenbaum A, Garniek A, Shemesh J, Fisman EZ, Stroh CI, Itzchak Y, et al. Dual-helical CT for detecting aortic atheromas as a source of stroke: comparison with transesophageal echocardiography. Radiology. 1998;208:153-8.

13. Shibata T, Hirai H, Fukui T, Aoyama T, Suehiro S. Assembly and deployment of a branched arch stent graft using the transaortic approach. Ann Thorac Surg. 2005;79:1790-2. 
14. Shiiya N, Asada M, Matsui Y, Sakuma M, Oba J, Gohda T, et al. Clinical results of selective cerebral perfusion during reconstruction of the transverse aortic arch. Nippon Kyobu Geka Gakkai Zasshi. 1994; 42:1858-64

15. Shiiya N, Kunihara T, Imamura M, Murashita T, Yoshiro M, Yasuda K. Surgical management of atherosclerotic aortic arch aneurysms using selective cerebral perfusion: 7-year experience in 52 patients. Eur J Cardiothorac Surg. 2000;17:266-71.

16. Safi HJ, Letsou GV, Iliopoulos DC, Subramaniam MH, Miller CC, Hassoun $\mathrm{H}$, et al. Impact of retrograde cerebral perfusion on ascending aortic and arch aneurysm repair. Ann Thorac Surg. 1997;63:1601-7.

17. Okita Y, Ando M, Minatoya K, Kitamura S, Takamoto S, Nakajima N. Predictive factors for mortality and cerebral complications in arteriosclerotic aneurysm of the aortic arch. Ann Thorac Surg. 1999;67:72-8.

18. Kieffer E, Richard T, Chiras J, Godet G, Cormier E. Preoperative spinal cord arteriography in aneurysmal disease of the descending thoracic and thoracoabdominal aorta: preliminary results in 45 patients. Ann Vasc Surg. 1989;3:34-46,

19. Williams GM, Perler BA, Burdick JF, Osterman FA Jr, Mitchell S, Merine D, et al. Angiographic localization of spinal cord blood supply and its relationship to postoperative paraplegia. J Vasc Surg. 1991;13: 23-33.

20. Ellozy SH, Carroccio A, Minor M, Jacobs T, Chae K, Cha A, et al. Challenges of endovascular tube graft repair of thoracic aortic aneurysm: midterm follow-up and lessons learned. J Vasc Surg. 2003;38: 676-83.

21. Khatibzadeh M, Mitusch R, Stierle U, Gromoll B, Sheikhzadeh A. Aortic atherosclerotic plaques as a source of systemic embolism. $J$ Am Coll Cardiol. 1996;27:664-9.

22. Tunick PA, Kronzon I. Atheromas of the thoracic aorta: clinical and therapeutic update. J Am Coll Cardiol. 2000;35:545-54.

23. White GH, May J, Waugh RC, Hughes C. Thoracic aortic aneurysms. In: Moore WS, Ahn SS, editors. Endovascular surgery. 3rd ed. Philadelphia: WB Saunders; 2001. p. 407-19.

24. Heltne JK, Koller ME, Lund T, Farstad M, Rynning SE, Bert JL, et al. Studies on fluid extravasation related to induced hypothermia during cardiopulmonary bypass in piglets. Acta Anaesthesiol Scand. 2001;45: 720-8.

25. Shamji MF, Maziak DE, Shamji FM, Ginsberg RJ, Pon R. Circulation of the spinal cord: an important consideration for thoracic surgeons. Ann Thorac Surg. 2003;76:315-21.

26. Marini CP, Levison J, Caliendo F, Nathan IM, Cohen JR. Control of proximal hypertension during aortic cross-clamping: Its effect on cerebrospinal fluid dynamics and spinal cord perfusion pressure. Semin Thorac Cardiovasc Surg. 1998;10:51-6.

27. Dardik A, Perler BA, Roseborough GS, Williams GM. Subdural hematoma after thoracoabdominal aortic aneurysm repair: an underreported complication of spinal fluid drainage? J Vasc Surg. 2002;36:47-50.
Appendix 1. Variables evaluated for logistic regression analysis to identify predictors for spinal cord injury

Preoperative variables

Age

Sex

Dissection

Emergency

History of aortic surgery

History of thoracic aortic aneurysm-dissection repair

History of infrarenal aortic aneurysm repair

Chronic obstructive pulmonary disease

History of cerebrovascular accident

Hypertension

Ischemic heart disease

Diabetes mellitus

Chronic renal failure

History of cigarette smoking

Maximum diameter of the aneurysm

Presence of severe atherosclerosis

Intraoperative variables

Concomitant total aortic arch replacement

Concomitant operation

Duration of circulatory arrest

Duration of aortic crossclamping

Duration of selective cerebral perfusion

Duration of cardiopulmonary bypass

Duration of operation

Intraoperative blood loss

Intraoperative transfusion

Axillary artery perfusion

Postoperative variables

Respiratory insufficiency

Acute renal failure

Stroke

Re-exploration for bleeding

Late cardiac tamponade

Duration of intensive care unit stay

Vertebral level of distal landing zone

Hospital mortality 